\title{
Development of Species-Specific PCR Primers for the Rapid and Simultaneous Identification of the Six Species of Genus Takifugu
}

\author{
Chun Mae Dong, Yeon Jung Park, Jae Koo Noh, Eun Soo Noh, Cheul Min An, \\ Jung-Ha Kang, Jung Youn Park, and ${ }^{\dagger}$ Eun-Mi Kim \\ Biotechnology Research Division, National Institute of Fisheries Science, Busan 46083, Korea
}

\begin{abstract}
Pufferfish (Takifugu spp.) are economically important edible marine fish. Mistakes in pufferfish classification can lead to poisoning; therefore, accurate species identification is critical. In this study, we used the mtDNA cytochrome c oxidase subunit I gene (COI) to design specific primers for six Takifugu species among the 21 domestic or imported pufferfish species legally sold for consumption in Korea. We rapidly and simultaneously identified these pufferfish species using a highly efficient, multiplex polymerase chain reaction (PCR) system with the six species-specific primers. The results showed that species-specific multiplex PCR (multiplex species-specific polymerase chain reaction; MSS-PCR) either specifically amplified PCR products of a unique size or failed. MSS-PCR yielded amplification fragment lengths of $897 \mathrm{bp}$ for Takifugu pardalis, $822 \mathrm{bp}$ for T. porphyreus, $667 \mathrm{bp}$ for T. niphobles, $454 \mathrm{bp}$ for T. poecilonotus, $366 \mathrm{bp}$ for T. rubripes, and $230 \mathrm{bp}$ for T. xanthpterus using the species-specific primers and a control primer (ca. 1,200 bp). We visualized the results using agarose gel electrophoresis to obtain accurate contrasts of the six Takifugu species. MSS-PCR analysis is easily performed and provides identification results within $6 \mathrm{~h}$. This technique is a powerful tool for the discrimination of Takifugu species and will help prevent falsified labeling, protect consumer rights, and reduce the risk of pufferfish poisoning.
\end{abstract}

Key words : Pufferfishes, Multiplex Species-Specific (MSS), Identification, PCR, Mitochondrial DNA COI

\section{INTRODUCTION}

Fish in the Tetraodontidae family are found in both freshwater and brackish waters of tropical and temperate coastal areas. Although many species in this family are economically important fishery resources, with unique flavors and high market value, they are often difficult to classify due to their wide variety of morphological variation (Kim \& Lee, 1990; Tyler, 1980). Within Tetraodontidae, most pufferfish species in the genus Takifugu are highly toxic to humans, containing tetrodotoxins that affect a variety of organs including the liver and ovaries. Toxicity varies considerably among regions and seasons; therefore, inaccurate Takifugu species classification carries a risk of tetrodotoxin poisoning. The Ministry of Food and Drug Safety of Korea allows the trade of only 21 domestic and imported pufferfish species; however, some of these species have very similar morphologies and are difficult to distinguish. Trade disputes arising from problems with species identification and classification among six Takifugu species commonly consumed in Korea have motivated the search for a simple, rapid, and effective molecular genetic

\footnotetext{
Manuscript received September 13, 2019, Received in revised form September 29, 2019, Accepted October 17, 2019

${ }^{\dagger}$ Corresponding Author : Eun-Mi Kim, Dr., Biotechnology Research Division, National Institute of Fisheries Science, Busan 46083, Korea. Tel: +82-51720-2462, E-mail: ocean0629@korea.kr
}

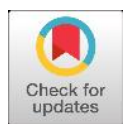

This is an Open Access article distributed under the terms of the Creative Commons Attribution Non-Commercial License (http:// creative-commons.org/licenses/by-nc/3.0) which permits unrestricted non-commercial use, distribution, and reproduction in any medium, provided the original work is properly cited. 
method for identification of these species.

Previous studies have applied morphological and component analyses to determine the species used in fishery products; recently, diagnostic polymorphic sites have been used to identify closely related species (Unseld et al., 1995; Kim et al., 2014). Molecular identification assays based on mtDNA sequences have been widely used to identify the origins of fishery resources and products for the prevention of fraud, mislabeling, and health risks (Rasmussen \& Morrissey, 2008; Cohen et al., 2009; Acar et al., 2017). However, little effort has been devoted to molecular-based species identification of Takifugu pufferfish species (Hsieh \& Hwang, 2004; Ishizaki et al., 2006; Hsieh et al., 2010; Luekasemsuk et al., 2015).

We developed a Takifugu species identification method that will contribute to the establishment of a safe fishery management and distribution system. We designed speciesspecific primers in the mtDNA cytochrome c oxidase subunit I (COI) gene region for the rapid and accurate identification of six Takifugu species, all of which are consumed in Korea: Takifugu pardalis, T. porphyreus, $T$. niphobles, T. poecilonotus, T. rubripes, and T. xanthpterus.

\section{MATERIALS AND METHODS}

For genetic analyses, we collected muscle tissue samples from $T$. pardalis $(\mathrm{n}=8), T$. porphyreus $(\mathrm{n}=28), T$. niphobles $(\mathrm{n}=8), T$. poecilonotus $(\mathrm{n}=6), T$. rubripes $(\mathrm{n}=23)$, and $T$. xanthpterus $(\mathrm{n}=198)$ stored by the National Institute of Fisheries Science (NIFS) and/or Marine Fish Resource Bank of Korea (MFRBK), and identified their morphological characteristics. These samples were collected in sterile tubes and preserved in $99.99 \%$ ethanol until DNA extraction. Total DNA from each sample was extracted using an automated DNA extraction system (MagExtractor MFX6100, Toyobo, Osaka, Japan). Genomic DNA was quantified using a spectrophotometer (Nanodrop ND-1000, Thermo Fisher Scientific, USA) and stored at $-20^{\circ} \mathrm{C}$ until genetic analyses.

We analyzed complete sequences of the mtDNA COI gene registered with the National Center for Biotechnology Information (NCBI) for the six pufferfish species: T. pardalis (GenBank accession no., AP009528.1), T. porphyreus (KY514076.1), T. niphobles (KY514069.1), T. poecilonotus (AP009539.1), T. rubripes (KP641572.1), and T. xanthpterus (KP641579.1) using the BioEdit v. 7.0.0 software to identify inter- and intra-species variation and conserved regions. Then we designed common primers (Taki-F35, TakiR1232) specific to the six species (Fig. 1A). Polymerase chain reaction (PCR) was performed in a total volume of $20 \mu \mathrm{L}$, comprising $2 \mu \mathrm{L}$ genomic DNA (20 ng), $0.6 \mu \mathrm{L}$ dNTP $(250 \mu \mathrm{M}), 2 \mu \mathrm{L} 1 \times$ PCR buffer containing $2 \mathrm{mM}$ $\mathrm{MgCl}_{2}, 0.4 \mu \mathrm{L} 10$ pmol forward primer, $0.4 \mu \mathrm{L} 10 \mathrm{pmol}$ reverse primer, $0.2 \mu \mathrm{L} 0.5 \mathrm{U}$ DNA Taq (Anti-HS Taq, TNT Research, Seoul, Korea). The primers used are listed in Table 1. PCR amplification was performed using an ABI 2720 Thermal Cycler under the following conditions: 10 min of initial denaturation at $95^{\circ} \mathrm{C} ; 37$ cycles of $45 \mathrm{~s}$ at $94^{\circ} \mathrm{C}, 45 \mathrm{~s}$ at $58^{\circ} \mathrm{C}$, and $1 \mathrm{~min}$ at $72^{\circ} \mathrm{C}$; and a final extension for $5 \mathrm{~min}$ at $72^{\circ} \mathrm{C}$. Amplified PCR products were sequenced using an ABI BigDye Terminator Cycle Sequencing Kit (ver. 3.1, Applied Biosystems, Foster, CA, USA), and analyzed using an ABI 3730XL DNA analyzer (Applied Biosystems).

We assembled forward and reverse sequences of the six Takifugu species with common primers using the SeqMan software (DNASTAR, USA), and searched for single nucleotide polymorphisms (SNPs) showing species specificity except for intraspecies point mutation. Then we designed species-specific forward primers for each target species in which the SNP was located at the 3' end (Fig. 2B(1)-(7), Table 1). We performed species-specific PCR analyses using the six forward primers (JB352, GB425, BS586, HJB796, JJB883, and GCB1028) and one reverse primer (Taki-R1232) under the PCR conditions described above for the common primer, but at an annealing temperature of 
Takifugu niphobles Takifugu poecilonotus Takifugu pardalis Takifugu porphyreus Takifugu rubripes Takifugu xanthopterus

Takifugu niphobles Takifugu poecilonotus Takifugu pardalis Takifugu porphyreus Takifugu rubripes Takifugu xanthopterus

Takifugu niphobles Takifugu poecilonotus Takifugu pardalis Takifugu porphyreus Takifugu rubripes Takifugu xanthopterus

Takifugu niphobles Takifugu poecilonotus Takifugu pardalis Takifugu porphyreus Takifugu rubripes Takifugu xanthopterus

Takifugu niphobles Takifugu poecilonotus Takifugu pardalis Takifugu porphyreus Takifugu rubripes Takifugu xanthopterus

Takifugu niphobles Takifugu poecilonotus Takifugu pardalis Takifugu porphyreus Takifugu rubripes Takifugu xanthopterus

Takifugu niphobles Takifugu poecilonotus Takifugu pardalis Takifugu porphyreus Takifugu rubripes Takifugu xanthopterus

Takifugu niphobles Takifugu poecilonotus Takifugu pardalis Takifugu porphyreus Takifugu rubripes Takifugu xanthopterus

Takifugu niphobles Takifugu poecilonotus Takifugu pardalis Takifugu porphyrews Takifugu rubripes Takifugu xanthopterus

Takifugu niphobles Takifugu poecilonotus Takifugu pardalis Takifugu porphyreus Takifugu rubripes Takifugu xanthopterus

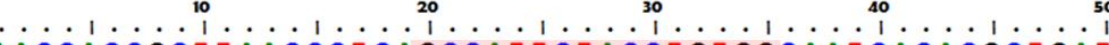

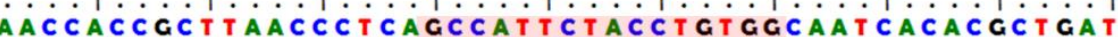
$A A C C A C C G C T T A A C C C T C A G C C A T T C T A C C T G T G G C A A T C A C A C G C T G A T$ AACCACCGCT TAACCCTCAGCCATTCTACCTGTGGCAATCACACGCTGAT AACCACCGCT TAACC CTCAGCCATTCTACCTGTGGCAATCACACGCTGAT AACCACCGCT TAACC CTCAGCCATTCTACCTGTGGCAATCACACGCTGAT AACCACCGCT TAACCCTCAGCCATTCTACCTCTGGCAATCACACGCTCAT (a) Taki-F35 100

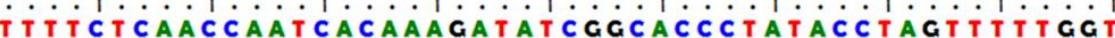
T T T T TCAACCAATCACAAAGATATCGGCACCCTATACCTACT TT T TGGT
$T T T T C T C A A C C A A T C A C A A A G A T A T C G G C A C C C T A T A C C T A C T T T T G G T$ T T T TCTCAACCAATCACAAAGATATCGGCACCCTATACCTAGTTTTTGGT T T T T T CAACCAATCACAAAGATATCGGCACC CTATACCTAGTTTTTGGT T T T T C T CAACCAAT CACAAAGATATCGGCACCCTATACCTAGTT T T T GGT

130

$$
140
$$

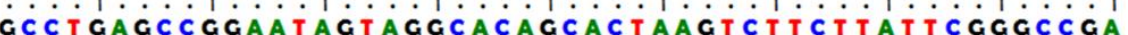
GCCTGAGCCGGAATAGTAGGCACAGCACTAAGTCTTCT TATTCGGGCCGA GCCTGAGCCGGAATAGTAGGCACTGCACTAAGTCTTCT TATTCGGGCCGA GC C T GAGCCGGAATAGTAGGCACAGCACTAAGTCT T CT TAT TCGGGCCGA GCCTGAGCCGGAATAGTAGGCACAGCACTAAGTCT TCT T AT TCGGGCCGA GC C T GAGCCGGAAT AGTAGGCACGGCACTAAGTCT TCT T AT T CGGGCCGA

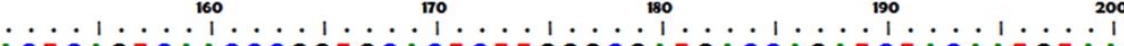

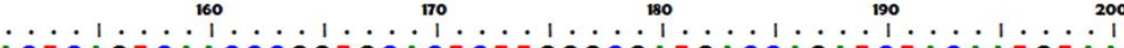
ACTCAGTCAACC CGGTGCACTCTTGGGCGATGACCAGATCTACAATGTAA ACTCAGTCAACCCGGCGCACTCTTGGGCGATGACCAGATTTACAATGTAA ACTCAGTCAACCCGGCGCACTCTTGGGCGATGACCAGATCTACAATGTAA $A C T C A G T C A A C C C G G C G C A T T A T T G G G C G A T G A C C A G A T C T A C A A T G T A A$ ACTCAGTCAACCCGGCGCACTCTTGGGCGATGACCAGATCTACAATGTAA ACT CAGTCAAC C CGGCGCACT C T T GGGCGATGACCAGAT T T ACAATGTAA

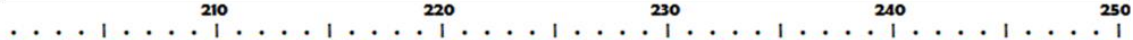

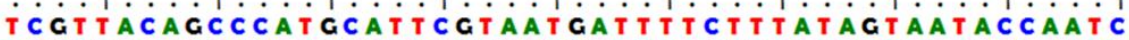
TCGTTACAGCCCATCCATTCGTAATGATTTTCTTTATAGTAATACCAATC TCGTTACAGCCCATGCATTCGTAATGATTTTCTTTATAGTAATACCAATC TCGTTACAGCC CATGCATTCGTAATAATT TTCTTTATAGTAATACCAATC TCGTTACAGCCCATGCATTCGTAATGATT T TCT T TATAGTAATACCAATC TCGT TACAGC C CATGCAT TCGTAATGAT T T T T T TATAGTAATACCAATC

$$
2602280 \quad 280 \quad 300
$$
ATG ATGAT TGGAGGCT T TGGAAACTGAT TAGT TCC C T TATAATTGGAGCCCC AT TAT T GGAGGCT T T GGGAACTGATTAAT TCC C C T TATAATCGGAGCC C C ATGATTGGAGGCTT TGGGAACTGACTAATTCCCCT TATAATCGGAGCCCC ATGATTGGAGGCT T TGGGAACTGATTAATCCCACT TATAATCGGAGCCCC ATGATTGGAGGCT T TGGGAACTGATTAGT TCCCCT TATAATCGGAGCCCC

$$
310
$$

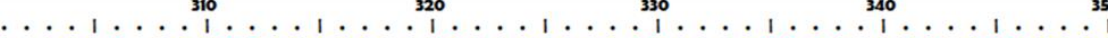

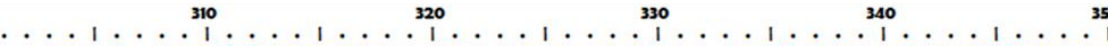
AGACATGGCCT TCC C C CGAATAAACAACATAAGCTTCTGACTGCTTCCCC AGACATGGCCTTCCCCCGAATAAACAACATAAGCTTCTGACTGCTTCCCC AGACATGGCCTTCCCCCGAATAAACAACATAAGCTTCTGACTACTTCCCC $A G A C A T G G C C T T C C C C C G A A T A A A C A A C A T A A G C T T C T G A C T A C T T C C C C$ $A G A C A T G G C C T$ T C C C C C AAT GAACAACATAACCTTCTGACTGCTTCC C AGACATGGCCT TCC CTCGAAT AAACAACATAAGCT TCTGACTGCT TCC C

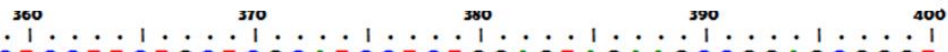

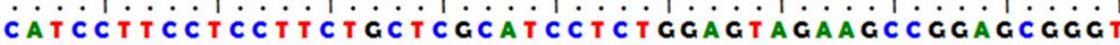
CATCCT T C CTCCT TCTACTCGCATCCTCTGGAGTAGAAGCCGGAGCGGGT CGTCCT TCCTCCTTCTGCTCGCATCCTCTGGAGTAGAAGCCGGAGCGGGT CATCCT T C C T C CT T CTACTCGCATCCTCTGGAGTAGAAGCCGGAGCGGGT CATCCT T C CTCCT TCT TCTCGCATCCTCTGGAGTAGAAGCCGGAGCGGGT CATCCT T C C T C T T C T GCTCGCATC C TCTGGAGTAGAAGCCGGAGCGGGT

(b) JB352

$$
410
$$
420 430 440

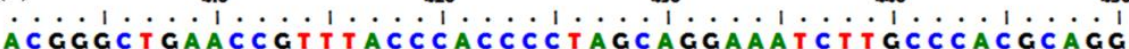
$A C G G G C T C A A C C G T T T A C C C A C C C C T A G C A G G A A A T C T T G C C C A C G C A G G$ $A C G G G C T G A A C C G T T T A C C C A C C C C T A G C A G G A A A T C T T C C C C A C G C A G G$ $A C G G G C T G A A C G G T T T A C C C A C C C T$ TAGCAGGAAATCT T GCCCACGCAGG ACGGGCTGAACTGTTTACCCACCCCTAGCAGGAAATCTTGCCCACGCAGG ACGGGCT GAACCGT T TACC CACC C T AGCAGGAAATCT T GCC CACGCAGG

$$
460
$$
470 (c) GB425 490 500

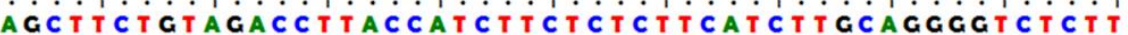
AGCTTCTGTAGACCTTACCATCTTCTCTCTCCATCTTCCAGGGGTCTCCT AGCTTCTGTAGACCTTACCATCTTCTCTCTTCATCTTCCAGGGGTCTCCT AGCTTCTGTAGACCTCACCATCTTCTCTCTTCATCT TGCAGGGGTCTCCT GGCTTCTGTAGAC CTCACCATCTTCTCTCTTCATCTTCCAGGGGTCTCCT AGCT TCTGTAGACCTTACCATCT TCTCTCTTCATCTTGCAGGGGTCTCCT

Fig. 1. Nucleotide alignment and information from the COI gene of six Takifugu species for use in species identification. Red boxes indicate the designed primer sets. COI, c oxidase subunit I. 
Takifugu niphobles Takifugu poecilonotus Takifugu pardalis Takifugu porphyreus Takifugu rubripes Takifugu xanthopterus

Takifugu niphobles Takifugu poecilonotus Takifugu pardalis Takifugu porphyreus Takifugu rubripes

Takifugu xanthopterus

Takifugu niphobles Takifugu poecilonotus Takifugu pardalis Takifugu porphyreus Takifugu rubripes Takifugu xanthopterus

Takifugu niphobles Takifugu poecilonotus Takifugu pardalis Takifugu porphyreus Takifugu rubripes Takifugu xanthopterus

Takifugu niphobles Takifugu poecilonotus Takifugu pardalis Takifugu porphyreus Takifugu rubripes Takifugu xanthopterus

Takifugu niphobles Takifugu poecilonotus Takifugu pardalis Takifugu porphyreus Takifugu rubripes Takifugu xanthopterus

Takifugu niphobles Takifugu poecilonotus Takifugu pardalis Takifugu porphyreus Takifugu rubripes Takifugu xanthopterus

Takifugu niphobles Takifugu poecilonotus Takifugu pardalis Takifugu porphyreus Takifugu rubripes Takifugu xanthopterus

Takifugu niphobles Takifugu poecilonotus Takifugu pardalis Takifugu porphyreus Takifugu rubripes Takifugu xanthopterus

Takifugu niphobles Takifugu poecilonotus Takifugu pardalis Takifugu porphyreus Takifugu rubripes

Takifugu xanthopterus

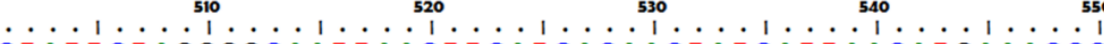
CTATTCTAGGGGCAATTAACT TCATCACAACTATCATTAACATGAAACCC CTATTCTAGGGGCAATCAACTTCATCACAACTATCATTAACATGAAACCC CTATTCTAGGGGCAATCAACT TCATTACAACTATCATTAACATGAAACCC CTAT TCTAGGGGCAATCAACT TCATCACAACTATCATTAACATGAAGCCC CTATTCTAGGGGCAATCAACT TCATCACAACTATCAT TAACATGAAGCCC CTAT TCTAGGGGCAATCAACT TCATCACAACTATCATTAACATAAAACCC

560 570 580 590 . 100 CCAGCAATCTCACAGTACCAAACACCTCTTTTCGTATCAGCCGTTTTAAT C CAGCAATCTCACAATAC CAAACACCCCTTTTCGTGTGAGCCGTCTTAAT C CAGCAATCTCACAATACCAAACACCTCTTT TCGTGTGAGCCGTTTTAAT C CAGCAATCTCACAATACCAAACACCTCTTTTCGTGTGAGCCGTTTTAAT C CAGCAATCTCACAATACCAAACACCTCT T T TCGTGTGAGCCGT T T T AAT C CAGCAATCTCACAATACCAAACACCTCTTT TCGTGTGAGCCGTT T TAAT

$$
\text { 610 }
$$
630 (d) BS586

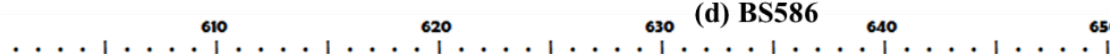
TACT GCT GTACT C CTCCTCCTCTCCCTTCCAGTCCTTGCAGCAGGGATTA TACT GCTGTACTTCTCCTGCTCTCCCTTCCAGTACT T GCAGCAGGGATTA TACTGCTGTACT TCTCCTGCTCTCCCT TCCAGTCCT TGCAGCAGGAAT TA TACTGCTGTACT TCTCCTGCTCTCCCTTCCAGTCCT TGCAGCAGGGAT TA TACTGCTGTACT TCTCCTGCTCTCCCT TCCAGTCCTTGCAGCAGGGATTA TACT GCTGTACT TCTCCT GCTCTCCCT TCCAGTCCT T GCAGCAGGGATTA

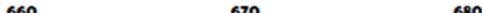

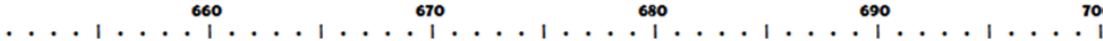
CAATGCT TCTCACTGACCGAAACTTAAATACAAC T TCT T T GAC C CAATACT TCTCACTGACCGAAACCTAAATACAACCTTCTTTGACCCAGCA CAAT ACT TCT CACT GACCGAAACCTGAATACAAC CT TCT T TGACC CAGCA CAATACT TCTCACTGACCGAAACCTAAATACAACCT TCT T TGACCCAGCA CAATACTTCTCACTGACCGAAACCTAAATACAACCTTCTTTGACCCAGCA CAATACT TCTCACTGACCGAAACCTAAATACAACCT TCT T TGACCCAGCA$$
720
$$$$
730
$$$$
740
$$

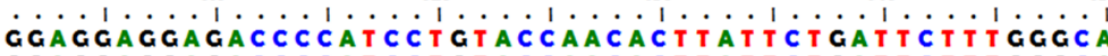
GGAGGAGGAGACCCCATCCTGTACCAACACT TATTCTGATTCTTTGGGCA GGAGGAGGAGACCCCATCCTGTACCAACACT TATTCTGATTCTTTGGGCA GGAGGAGGAGACCCCATCCTGTACCAACACT TATTCTGATTCTTCGGGCA GGAGGAGGAGACCCCATCTTGTACCAACACT TATTCTGATTCTTTGGACA GGAGGAGGAGACC C CATCCTGTACCAACACT TAT TCTGAT TCT T TGGGCA

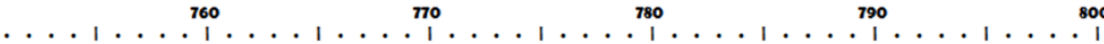

770

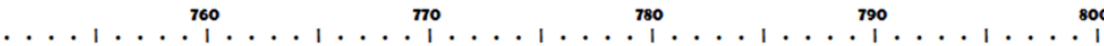

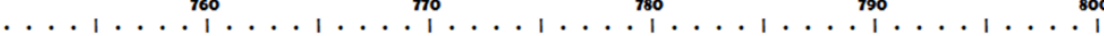
C C CTGAAGT T TACATTCTAATTCTCCCTGGCTTCGGGATGATT TCACATA C C C T GAAGTCTACATTCTAAT TCTCCCCGGCT T CGGAATAATCTCGCACA C C CTGAAGTCTACATTCTAATTCTCCCTGGCT TCGGGATAATCTCACACA C C CTGAAGTCTATAT TCTGATTCTCCCTGGCT TCGGGATAATT TCACATA C C CTGAAGTCTACATTCTAATTCTCCCTGGCTTCGGAATAATT TCACACA C C C T GAAGTCTACAT T C TAAT TCTCC CTGGCT TCGGGATAAT T T CACACA

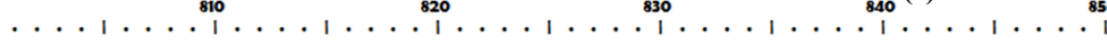

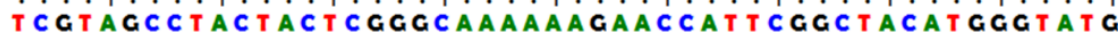
TCGTAGCCTACTACT CGGGCAAAAAGGAACCATTCGGTTACATGGGCATC T TGTAGCCTACTACTCGGGCAAAAAGGAACCATTCGGCTATATGGGCATG TCGTAGCCTACTACTCGGGCAAAAAAGAACCATTCGGCTACATGGGTATG TCGTAGCCTACTACTCGGGCAAAAAAGAACCATTCGGCTACATGGGCATC TCGTAGCCTACTACTCGGGCAAAAAAGAACCATTCGGT TATATGGGCATG

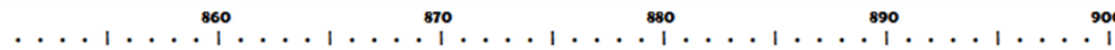

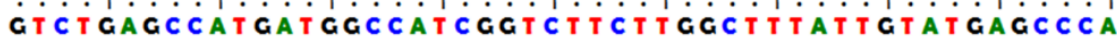
GTATGAGCCATGATGGCCATCGGTCTTCTTGGCTT TATTGTATGAGCCCA GTCTGAGCCATGATGGCCATCGGTCTTCTTGGCTTTATTGTATGAGCCCA GTCTGAGCCATGATGGCCATCGGCCT TCT TGGCT T T AT T GTATGAGCCCA GTCTGAGCCATAATGGCCATCGGTCTTCTTGGTTTTATTGTATGAGCCCA GTCTGAGCCATGATGGCCATCGGTCTTCTTGGCT T TAT TGTATGAGCCCA

$$
910
$$

930 (f) JJB883

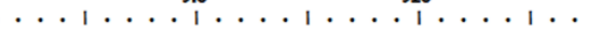

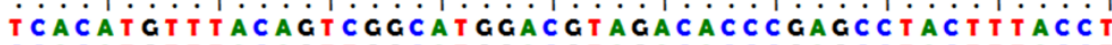
C CACATGTT TACAGT TGGCATGGACGTAGACACC CGAGCCTACT T T ACCT TCACATGTTTACAGTTGGCATGGACGTAGACACCCGAGCCTACTTTACCT TCACATGTT TACAGTCGGCATGGACGTAGACACCCGAGCCTACT T TAC C T C CACATGTT TACAGTCGGCATGGACGTAGACACCCGAGCCTACT T TACCT TCACATGTT TACAGTCGGTATGGACGTAGACACCCGAGCCTACT T ACC

960 970 980 990 1000

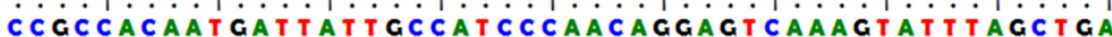
CCGCCACAATAATTATTGCCATCCCAACAGGGGTAAAAGTATTCAGTTGA C CGCCACAATAATTATCGCTATCCCGACAGGAGTCAAAGTATTTAGCTGA C CGCCACAATAAT TAT TGCCATCCCAACAGGAGTCAAAGTAT T TAGCTGA CTGCCACAATAATTATTGCCATCCCGACAGGAGTCAAAGTATT TAGCTGA C CGCCACAATAAT TAT TGCCATTCCAACAGGAGTCAAAGTAT T TAGCTGA

Fig. 1. Continued. 
Takifugu niphobles Takifugu poecilonotus Takifugu pardalis Takifugu porphyreus Takifugu rubripes Takifugu xanthopterus

Takifugu niphobles Takifugu poecilonotus Takifugu pardalis Takifugu porphyreus Takifugu rubripes Takifugu xanthopterus

Takifugu niphobles Takifugu poecilonotus Takifugu pardalis Takifugu porphyreus Takifugu rubripes Takifugu xanthopterus

Takifugu niphobles Takifugu poecilonotus Takifugu pardalis Takifugu porphyreus Takifugu rubripes Takifugu xanthopterus

Takifugu niphobles Takifugu poecilonotus Takifugu pardalis Takifugu porphyrews Takifugu rubripes

Takifugu xanthopterus

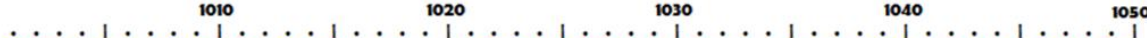
CTTGCAACCTTGCATGGAGGATCAATTAAATGAGAAACCCCTATACT ATG CT TGCAACCT TGCATGGAGGATCAAT TAAATGAGAAACCCCTATACTATG CT TGCAACCTTGCATGGAGGATCAAT TAAATGAGAAACCCCTATACTATG CT TGCAACCT TGCATGGAGGATCAAT TAAATGAGAAAC C C T TATACTATG CT TGCAACCTTGCATGGAGGATCAAT TAAATGAGAAACCCCTATACTATC CTCGCAACCTTACATGGGGGCTCAATTAAATGAGAAACCCCTATACTATG

$\ldots{ }^{1060} \ldots 0^{1070} \ldots \stackrel{10}{10}^{1090} \ldots 1 \ldots 0^{100}$ GGCCCTCGGCTTCATCTTCCTATTTACAGTGGGTGGCCTAACCGGAATTC GGCCCTCGGCTTCATCTTCCTATT TACAGTGGGTGGCCTAACCGGAATTC AGCCCTCGGCTTCATCTTCCTATT TACAGTGGGTGGCCTAACCGGAATTC AGCCCTCGGCTTCATCTTCCTATT TACAGTGGGTGGCCTAACCGGAATTG AGCCCTCGGCTTCATCTTCCTATTTACAGTGGGTGGCCTAACCGGAATTG AGCCCTCGGCTTCATCTTCCTATTTACAGTGGGTGGCCTAACCGGAATTG mo 1120 $11120 \quad 1130 \quad 1150$ mo $11120 \quad 1130 \quad 1150$ TCCTGGCCAACTCATCCCTAGACATTGTGTTACACGACACCTACTACGTA TCCTGGCCAACTCATCCCTAGACATTGTGT TACACGACACCTACTACGTA TCCTAGCCAACTCATCCCTAGACATTGTATTACACGACACCTACTACGTA TCCTGGCCAACTCATCCCTAGACATTGTGTTACACGACACCTACTACGTA TCCTAGCCAATTCATCCCTAGACATCGTATTACACGACACCTACTACGTA TCCTAGCCAACTCATCCCTAGACATTGTATTACACGACACCTACTACGTA

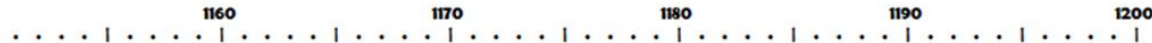

GTTGCCCACTTCCACTATGTCCTTTCCATGGGTGCTGTATTTGCAATTAT GTTGCCCATTTCCACTATGTCCTCTCCATGGGTGCTGTATTTGCAATCAT GTTGCCCATTTCCACTATGTCCTCTCCATGGGTGCTGTATTTGCAATTAT GTTGCCCATT TCCACTATGTCCTTTCCATGGGTGCTGTGTTTGCAAT TAT GT TGCCCATTTCCACTACGTCCTCTCCATGGGTGCTGTATT TGCAAT TAT GT TGCCCACT TCCACTATGTCCTCTCCATGGGTGCTGTATTTGCAATTAT GT T GC C C AC T T C $\ldots 12201230$ GGGTGCATTCGTGCACTGATTCCCACTATTTT GGGTGCATTCGTACACTGATTCCCACTATTCT GGGCGCATTCGTGCACTGATTCCCACTATTTT GGGTGCATTCGTACACTGGTTCCCACTATTTT GGGTGCATTCGTACACTGATTCCCACTATTTT GGGT GCATTCGTGCACTGATTCCCACTATTCT

(a) Taki-R1232

Fig. 1. Continued.

$60^{\circ} \mathrm{C}$. Amplified PCR products were subjected to $2 \%$ agarose gel electrophoresis $(100 \mathrm{~V}, 40 \mathrm{~min})$ with $1 \times$ RedSafe (iNtRon, Korea), and confirmed using the Gel Doc image analysis system (ATTO Corp., Japan).

\section{RESULTS AND DISCUSSION}

A region of the cytochrome COI is widely applied in genetic identification of animals including fishes and
(A) common primer
(1)
(2) (3)
(4)
(5) (6)

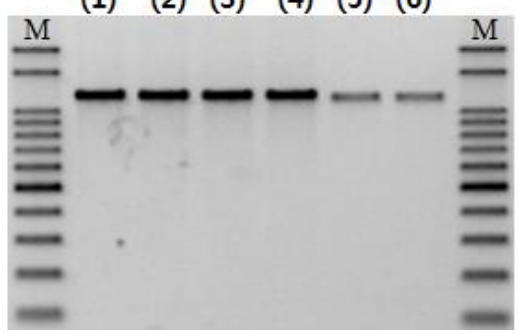

(B) species-specific primers

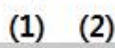

\section{(3)}

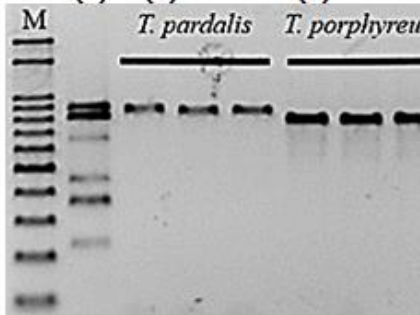

(5)

T. niphobles
(7)

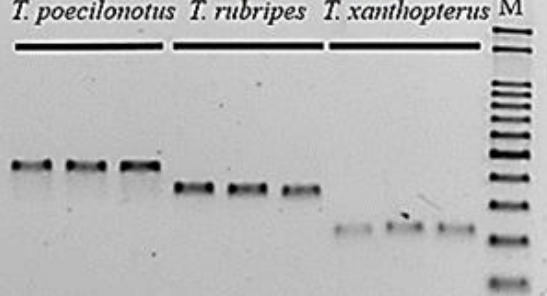

Fig. 2. Identification of species by multiplex polymerase chain reaction (PCR) using common primer and speciesspecific primers. Samples are identified as follows: (A) common primer; (1) T. pardalis, (2) T. porphyreus, (3) T. niphobles, (4) T. poecilonotus, (5) T. rubripes, (6) T. xanthopterus, (B) lane (1) template mixture, (2) T. pardalis, (3) T. porphyreus, (4) T. niphobles, (5) T. poecilonotus, (6) T. rubripes, (7) T. xanthopterus, (M) 100 bp DNA ladder (iNtRON, Korea). 
Table 1. Species-specific primers used for multiplex polymerase chain reaction (PCR)

\begin{tabular}{cccc}
\hline \hline Primers & Sequence $\left(5^{\prime} \rightarrow 3^{\prime}\right)$ & Product size $(\mathrm{bp})$ & Specific species \\
\hline JB352 & TTCTGACTACTTCCCCCG & 897 & Takifugu pardalis \\
GB425 & ACGGTTTACCCACCCT & 822 & T. porphyreus \\
BS586 & GTACCAAACACCTCTTTTCGTA & 667 & T. niphobles \\
HJB796 & CGGCTTCGGAATAATCTCG & 454 & T. poecilonotus \\
JJB883 & GCCATCGGTCTTCTTGGT & 366 & T. rubripes \\
GCB1028 & TCGCAACCTTACATGGG & 230 & T. xanthpterus \\
Taki-F35 & GCAATCACACGCTGATT & & Common primer \\
Taki-R1232 & AYAATAGTGGGAARCAGTG & 1,197 & (Genus Takifugu $)$ \\
\hline
\end{tabular}

improved effectiveness in species identification on the previous report (Rasmussen \& Morrissey, 2008; Cohen et al., 2009; Acar et al., 2017). PCR analyses using speciesspecific primers have excellent sensitivity and specificity, and can analyze large numbers of samples within a short time. These methods are widely used for species identification because they are simpler than DNA sequence analyses (Hwang et al., 2002). Also, MSS-PCR methods are economical in terms of time, effort, and cost compared to other DNA based methods, and the determination of the most important species can provide clear and repeatable results (Sezaki et al., 2005; Noh et al., 2017).

We successfully developed and applied a multiplex PCR assay based on species-specific variation for rapid and simultaneous identification of six target species: T. pardalis, T. porphyreus, T. niphobles, T. poecilonotus, T. rubripes, and T. xanthpterus. This is the first study to use conventional PCR amplification to identify six pufferfish species commonly imported into the Korean fishery market.

We obtained 1,252 bp sequences of the mtDNA COI region from the six species, and used a 1,197 bp common primer to confirm MSS-PCR amplification (Fig. 1). Sequences obtained using the common primer were analyzed using the DNA Sequence Polymorphism (DnaSP) v. 5.10.01 software. Haplotype analysis results for a total of 271 individuals among the six species indicated 4 haplotypes of $T$. pardalis $(\mathrm{n}=8), 4$ of $T$. porphyreus $(\mathrm{n}=28), 7$ of $T$. niphobles $(\mathrm{n}=8), 4$ of $T$. poecilonotus $(\mathrm{n}=6), 6$ of $T$. rubripes $(\mathrm{n}=23)$, and 32 of $T$. xanthpterus $(\mathrm{n}=198)$. Except for intraspecies genetic variation, species-specific SNPs were observed at $352 \mathrm{bp}$ (JB352), $425 \mathrm{bp}$ (GB425), $586 \mathrm{bp}$ (BS586), 796 bp (HJB796), 883 bp (JJB883), and 1,028 bp (GCB1028) (Fig. 1).

MSS-PCR products containing the same amounts of forward primers were confirmed by agarose gel electrophoresis, with accurate DNA amplification of each species and clear distinction among amplified products by size (Table 1). Sequencing analyses confirmed that MSSPCR products of the six species had $100 \%$ identity with the expected regions. Primer dimers and nonspecific amplification products were not observed, and no crossreactions were observed among species-specific amplifications in DNA mixtures of the six species (Fig. 2). The MSS-PCR assay sensitivity test showed that DNA template concentrations of stored products from the six species were $10 \mathrm{ng} / \mu \mathrm{L}, 1 \mathrm{ng} / \mu \mathrm{L}, 0.1 \mathrm{ng} / \mu \mathrm{L}$, and 0.01 $\mathrm{ng} / \mu \mathrm{L}$. The PCR assay sensitivity of each species was detectable to a concentration of $1 \mathrm{ng} / \mu \mathrm{L}$ among all six 


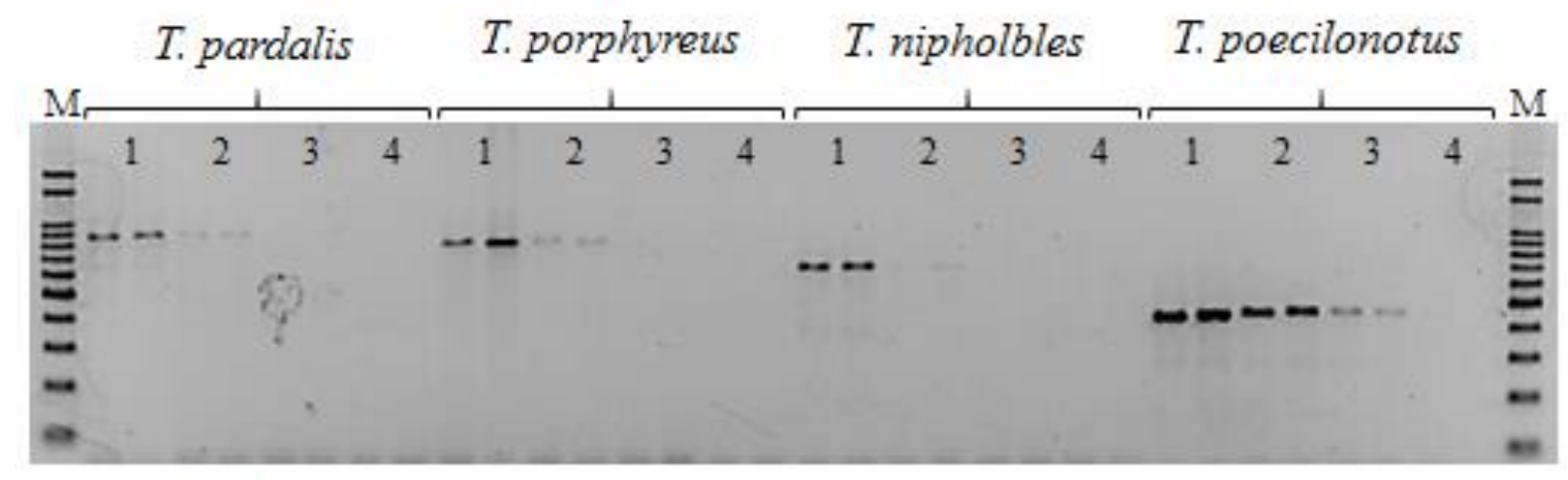

\section{T. rubripes $\quad$ T. xanthopterus}

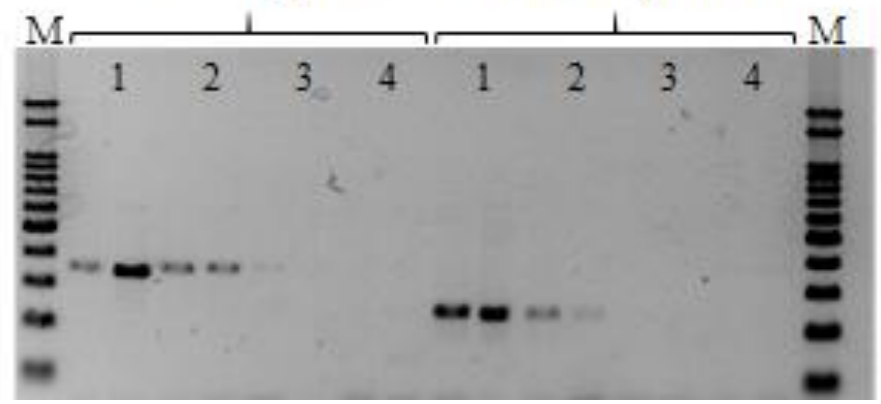

Fig. 3. Sensitivity for detection of the six Takifugu species using species-specific primer sets. Sensitivity analyses were performed using 10-fold amplification with serial dilution from 10 to $0.01 \mathrm{ng} / \mu \mathrm{L}$ genomic DNA from each of two individuals. Samples are identified as follows: lane (M) 100 bp DNA ladder (iNtRON, Korea). (1) $10 \mathrm{ng}$, (2) $1 \mathrm{ng}$, (3) $0.1 \mathrm{ng}$, (4) $0.01 \mathrm{ng}$.

species (Fig. 3).

In conclusion, the molecular method developed in this study was simple, rapid, and inexpensive compared to direct sequencing analyses, and did not require highquality equipment. The MSS-PCR method is capable of clearly distinguishing and/or authenticating six Takifugu species in case of mislabeling via accident or fraud. This technique can be used as a regulatory tool to protect public health and enforce Korean fishery product import regulations.

\section{ORCID}

Chun Mae Dong

https://orcid.org/0000-0001-7068-5939

Yeon Jung Park

https://orcid.org/0000-0001-5363-7847
Jae Koo Noh

https://orcid.org/0000-0002-9010-6225

Eun Soo Noh

https://orcid.org/0000-0003-3880-5050

Cheul Min An

https://orcid.org/0000-0002-5033-2498

Jung-Ha Kang

https://orcid.org/0000-0002-6554-4581

Jung Youn Park

https://orcid.org/0000-0002-6948-6059

Eun-Mi Kim

https://orcid.org/0000-0003-3376-2995 


\section{CONFLICT OF INTEREST}

The authors declare no potential conflict of interest.

\section{ACKNOWLEDGEMENTS}

This work was partially supported by the National institute of Fisheries Science (R2019030) "Screening and utilization of fisheries genetic resources".

\section{AUTHOR CONTRIBUTIONS}

Conceptualization: Kim EM.

Data curation: Park YJ, Noh ES.

Formal analysis: Noh JK, Park YJ, Dong CM.

Methodology: Kim EM, Park YJ, Dong CM.

Software: Park YJ, Dong CM.

Validation: Kim EM, Kang JH, An CM.

Investigation: Kim EM, Park JY.

Writing - original draft: Kim EM, Dong CM.

Writing - review \& editing: Kim EM, Park YJ, Dong CM.

\section{ETHICS APPROVAL}

This article does not require IRB/IACUC approval because there are no human and animal participants.

\section{REFERENCES}

Acar C, Ishizaki S, Nagashima Y (2017) Toxicity of the lessepsian pufferfish Lagocephalus sceleratus from eastern Mediterranean coasts of Turkey and species identification by rapid PCR amplification. Eur Food Res Technol 243:49-57.

Cohen NJ, Deeds JR, Wong ES, Hanner RH, Yancy HF, White KD, Thompson TM, Wahl M, Pham TD, Guichard FM, Huh I, Austin C, Dizikes G, Gerber SI
(2009) Public health response to puffer fish (tetrodotoxin) poisoning from mislabeled product. J Food Prot 72:810817.

Hsieh YW, Hwang DF (2004) Molecular phylogenetic relationships of puffer fish inferred from partial sequences of cytochrome $b$ gene and restriction fragment length polymorphism analysis. J Agric Food Chem 52:4159-4165.

Hsieh CH, Chang WT, Chang HC, Hsieh HS, Chung YH, Hwang DF (2010) Puffer fish-based commercial fraud identification in a segment of cytochrome $b$ region by PCR-RFLP analysis. Food Chem 121:1305-1311.

Hwang CY, Youn HY, Han HR (2002) Development of non-invasive fecal PCR assay for detecting the Helicobacter species infection in dogs. J Vet Clin 19:295298.

Ishizaki S, Yokoyama Y, Oshiro N, Teruya N, Nagashima Y, Shiomi K, Watabe S (2006) Molecular identification of pufferfish species using PCR amplification and restriction analysis of a segment of the 16S rRNA gene. Comp Biochem Phys D Genom Proteom 1:139-144.

Kim IS, Lee WO (1990) Synopsis of the suborder Tetraodontoidei (Pisces; Tetraodontiformes) from Korea. Korean J Ichthyol 2:1-27.

Kim KH, Lee HY, Kim YS, Kim MR, Jung YK, Lee JH, Chang HS, Park YC, Kim SY, Choi JD, Jang YM (2014) Development of species-specific PCR to determine the animal raw material. J Food Hyg Saf 29:347-355.

Luekasemsuk T, Panvisavas N, Chaturongakul S (2015) TaqMan qPCR for detection and quantification of mitochondrial DNA from toxic pufferfish species. Toxicon 102:43-47.

Noh ES, Lee MN, Kim EM, Park JY, Noh JK, An CM, Kang JH (2017) Development of a multiplex PCR assay for rapid identification of Larimichthys polyactis, L. crocea, Atrobucca nibe, and Pseudotolithus elongates. J Life Sci 27:746-753. 
Rasmussen RS, Morrissey MT (2008) DNA-based methods for the identification of commercial fish and seafood species. Comp Rev Food Sci Food Saf 7:280-295.

Sezaki K, Itoi S, Watabe S (2005) A simple method to distinguish two commercially valuable eel species in Japan Anguilla japonica and A. anguilla using polymerase chain reaction strategy with a species-specific primer. Fish Sci 71:414-421.
Tyler JC (1980) Osteology, phylogeny and higher classification of the fishes of the order plectognathi (Tetraodontiformes). NOAA Tec Rep NMFS Cir 434:422.

Unseld M, Beyermann B, Brandt P, Hiesel R (1995) Identification of the species origin of highly processed meat products by mitochondrial DNA sequences. PCR Methods Appl 4:241-243. 